INPLASY

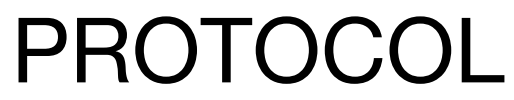

To cite: Yin et al. Vibration training to relieve delayed muscle soreness: A metaanalysis. Inplasy protocol 2021120115. doi:

10.37766/inplasy2021.12.0115

Received: 25 December 2021

Published: 25 December 2021

Corresponding author:

Yin Yikun

1365344195@qq.com

Author Affiliation:

Guangxi Normal University.

Support: Not Reported.

Review Stage at time of this submission: Data analysis.

Conflicts of interest:

None declared.

\section{Vibration training to relieve delayed muscle soreness: A meta-analysis}

Review question / Objective: This paper systematically evaluates the effect of VT on DOMS mitigation by Metaanalysis to provide an updated evidence-based basis.

Condition being studied: Vibration training to relieve delayed muscle soreness.

Eligibility criteria: (i) age > 18 years, good physical condition, no contraindications to exercise; (ii) induction of DOMS by exercise; (iii) no lower limb muscle pain and musculoskeletal disorders, no neurological and cardiovascular diseases.

INPLASY registration number: This protocol was registered with the International Platform of Registered Systematic Review and Meta-Analysis Protocols (INPLASY) on 25 December 2021 and was last updated on 25 December 2021 (registration number INPLASY2021120115).

\section{INTRODUCTION}

Review question / Objective: This paper systematically evaluates the effect of VT on DOMS mitigation by Meta-analysis to provide an updated evidence-based basis.

Condition being studied: Vibration training to relieve delayed muscle soreness.

\section{METHODS}

Participant or population: i) age $>18$ years, good physical condition, no contraindications to exercise; (ii) induction of DOMS by exercise; (iii) no lower limb muscle pain and musculoskeletal disorders, no neurological and cardiovascular diseases. 
Intervention: Vibration training.

Comparator: Stretching, massage, or no intervention.

Study designs to be included: Randomized controlled trial (RCT).

Eligibility criteria: (i) age > 18 years, good physical condition, no contraindications to exercise; (ii) induction of DOMS by exercise; (iii) no lower limb muscle pain and musculoskeletal disorders, no neurological and cardiovascular diseases.

Information sources: China Knowledge Network (CNKI), VIP, PubMed, EBSCO, and Web Of Science.

Main outcome(s): Visual analogue scale (VAS), pressure pain threshold (PPT), serum creatine kinase (CK), lactate dehydrogenase (LDH) and knee mobility (ROM).

Quality assessment / Risk of bias analysis: Cochrane Handbook for Systematic Reviews of. Interventions.

Strategy of data synthesis: RveMan 5.4.

Subgroup analysis: Different test time.

Sensitivity analysis: RveMan $\mathbf{5 . 4 .}$

Country(ies) involved: China.

Keywords: vibration training; delayed muscle soreness; subjective pain; pressure pain threshold; serum creatine kinase; Meta-analysis.

Contributions of each author:

Author 1 - YIN YIKUN - drafted the manuscript; data curation.

Email: 1365344195@qq.com

Author 2 - WANG JIALIN - The author provided statistical expertise.

Email: 1003723595@qq.com

Author 3 - SUN JUNZHI - The author contributed to the development of the selection criteria, and the risk of bias assessment strategy. 